The AstrophysicAL Journal, 506:341-346, 1998 October 10

(C) 1998. The American Astronomical Society. All rights reserved. Printed in U.S.A.

\title{
PULSAR RADIO EMISSION BY CONVERSION OF PLASMA WAVE TURBULENCE: NANOSECOND TIME STRUCTURE
}

\author{
JAMES C. WEATHERALL \\ Department of Physics, New Mexico Institute of Mining and Technology, Socorro, NM 87801 \\ Received 1997 June 27; accepted 1998 May 14
}

\begin{abstract}
The plasma wave turbulence emission mechanism is studied to make predictions for the temporal characteristics of pulsar radio emission. The turbulent emission process consists of a cycle of electrostatic wave growth and modulational conversion into radiative modes. The onset of plasma wave turbulence is marked by explosive spatial collapse of regions of high electric field and bursts of radiation. Intrinsic time structure is found on subnanosecond to $10 \mathrm{~ns}$ timescales. The pulse exhibits an unusual spectral signature because of the coupling of the electromagnetic modes to the underlying electrostatic pump wave. This prediction can be tested with ultrashort timescale observations.

Subject headings: instabilities — plasmas — pulsars: general — radiation mechanisms: nonthermal
\end{abstract}

\section{INTRODUCTION}

Radio microstructure of pulsars is the extremely fine temporal fluctuation observed at less than $1 \mu \mathrm{s}$, and it has long been regarded as caused by inhomogeneity or temporal evolution of the source. High time resolution observations (Hankins \& Weatherall 1998; Moffet 1997) are providing new data on timescales shorter than $10 \mathrm{~ns}$. This very short time structure - which might be called nanostructure - is indicative of the dynamical behavior of the coherence process underlying the emission.

This paper explores a radio emission model based on the conversion of plasma turbulence, which is one of several prominent candidates for the pulsar emission mechanism (see, for example, reviews by Asseo 1996 and Melrose 1996). This theory relates closely to a specific emission process proposed by Asseo et al. (1990), and modified by Weatherall (1997). The computational treatment in this paper will provide solutions for temporal behavior in greater detail than attempted in previous efforts to link pulsar microstructure to plasma turbulence processes (Arons 1981; Chian \& Kennel 1983; Mofiz, de Angelis, \& Fortani 1985; Chian 1992; Asseo 1993; see also Gangadhara, Krishan, \& Shukla 1993). The model derives from an analysis of coupled mode equations describing the evolution of plasma wave turbulence in strongly magnetized pair plasma. The short time structure inferred from the emission theory provides theoretical predictions to test against future observational data.

\section{COUPLED MODAL EQUATIONS DESCRIBING PLASMA TURBULENCE}

The full derivation of this model is given in Weatherall (1997). In the computational model, the electric field in the polar cap plasma is a linear superposition of wavemodes of amplitude $A_{m n}$ :

$$
\begin{aligned}
\boldsymbol{E}(\boldsymbol{x}, t)= & \frac{1}{2} \sum_{m=-\infty}^{+\infty} \sum_{n=-\infty}^{+\infty} A_{m n}(t) \hat{\boldsymbol{e}}_{m n} e^{i \omega_{p} t-i k_{m} x_{\|}-i k_{n} x_{\perp}} \\
& + \text { complex conjugate } .
\end{aligned}
$$

The pair plasma frequency is $\omega_{p}=\left(8 \pi n e^{2} / m_{e}\right)^{1 / 2}$, where $n$ is the electron number density. In the source volume of size $L$, the spatial variations are limited to the direction $x_{\|}$parallel to the magnetic field and to one direction $x_{\perp}$ perpendicular to the magnetic field. Although a two-dimensional turbu- lence might be anticipated in the form of filamentary structure in $(r, \phi)$ cylindrical coordinates, rectangular coordinates are used here for computational simplicity and are assumed to preserve the dimensional characteristics of the turbulence. The wavevector $\boldsymbol{k}_{m n}$ has wavenumber components $k_{m}=2 \pi m / L$ and $k_{n}=2 \pi n / L$.

The turbulence is modeled as ordinary branch wavemodes (see, e.g., Arons \& Barnard 1986), where the natural frequencies of the waves of interest are assumed to be near the pair plasma frequency and are given explicitly by $\omega_{m n}^{2}=$ $k_{m}^{2} c^{2}+(Y+D) / 2$. The polarization vectors of these waves, $\hat{\boldsymbol{e}}_{m n}$, have components perpendicular and parallel to the field in the ratio $X /(Y+D)$. Near the plasma frequency, the polarizations are predominantly along the magnetic field direction and couple strongly to the plasma. The quantities $X, Y$, and $D$ are defined as $X=2 k_{m} k_{n} c^{2}, Y=\omega_{p}^{2}-$ $k_{m}^{2} c^{2}+k_{n}^{2} c^{2}+3 k_{m}^{2} \bar{v}_{\|}^{2}$, and $D=\left(X^{2}+Y^{2}\right)^{1 / 2}$. The velocity parameter $\bar{v}_{\|}^{2}$ is the sum of the squared velocity moments of the electron and positron velocity distributions, and is assumed to be nonrelativistic: the numerical value adopted here is $\bar{v}_{\|}=0.26 c$. The limit of infinite magnetic field is used in this formulation.

The time-dependent evolution of the wave amplitudes is given by coupled equations

$$
\left(2 i \omega_{p} \frac{\partial}{\partial t}+\omega_{m n}^{2}-\omega_{p}^{2}\right) A_{m n}=c_{m n} \Lambda_{m n}^{(\mathrm{NL})} .
$$

The mode coupling is caused by variations in the refractive index induced by ponderomotive effects in the plasma. The coupling is cubic in the field. The right-hand side of the modified wave equation is the Fourier series component of the nonlinear coupling term, which can be written as a convolution

$$
\begin{aligned}
\Lambda_{m n}^{(\mathrm{NL})} & =\frac{\omega_{p}^{2}}{8 \pi m n \bar{v}_{\|}^{2}} \sum_{1,2,3} A_{m_{1} n_{1}} A_{m_{2} n_{2}} A_{m_{3} n_{3}}\left(\hat{\boldsymbol{e}}_{m_{1} n_{1}} \cdot \hat{\boldsymbol{e}}_{\|}\right) \\
& \times\left(\hat{\boldsymbol{e}}_{m_{2} n_{2}} \cdot \hat{\boldsymbol{e}}_{\|}\right)\left(\hat{\boldsymbol{e}}_{m_{3} n_{3}} \cdot \hat{\boldsymbol{e}}_{\|}\right) \delta_{m-m_{2}+m_{1}-m_{3}} \delta_{n-n_{2}+n_{1}-n_{3}} .
\end{aligned}
$$

Here the sum includes the range of all wavenumber indices $m_{1}, n_{1}, m_{2}, n_{2}, m_{3}, n_{3}$, excluding $m_{1}=m_{2}$ and $n_{1}=n_{2}$. In the spectral method numerical algorithm, this sum is evaluated by forming the product in $\left(x_{\|}, x_{\perp}\right)$ space and then computing the finite Fourier transform to wavenumber space, $\left(k_{m}, k_{n}\right)$. Because only wave field components parallel to the background magnetic field generate currents in the 
plasma, the coupling includes the geometric factor $c_{m n}=$ $\left|\hat{\boldsymbol{e}}_{m n} \cdot \hat{\boldsymbol{e}}_{\| \mid}\right|$.

The computer model uses a finite Fourier series of $64 \times 64$ terms. (Because some of the mode amplitudes are aliased to zero, the actual system consists of $42 \times 42$ modes.) The electric field on a $64 \times 64$ spatial grid is constructed through a Fourier sum, i.e., the inverse transform.

The assumptions used in deriving the modal equations include wave frequencies $\omega_{m n}$ near $\omega_{p}$; separable timescales, $\partial A_{m n} / \partial t \ll \omega_{p} A_{m n} ;$ quasi-neutrality; adiabatic (inertialess) low-frequency motions; and particle motions strictly along the magnetic field (large field limit).

The simulation volume is limited to a localized region within the polar cap plasma. In this work, the length, $L$, of the grid corresponds to the transverse extent of the polar cap plasma, which is confined to the region having magnetic field lines extending beyond the light cylinder. $L$ is much smaller than the neutron star radius (especially when Lorentz contraction in the direction of the flow is taken into account), and during the course of a solution, the simulation volume moves a distance in the star frame much less than the density-scale height. The local plasma frequency derives from the unperturbed plasma density, which is assumed to be the same everywhere within the simulation volume. There is no bulk flow or flow gradients present in the simulation rest frame.

It will be shown in the next section that emission occurs in temporally transient and spatially localized structures associated with turbulence, and is narrow line in frequency. The observed broadband radio spectra would be a superposition of many narrow-line emissions from different locations in the outflowing pair plasma. The local plasma frequency varies over many orders of magnitude in the polar cap current flow, as inferred observationally from radius-to-frequency mapping. The emission of individual bursts should be uncorrelated (i.e., shot noise), since the radiating structures are spatially unconnected. The emission can occur throughout the current flow because the underlying two-stream instability is a global response of the pair plasma to the electrodynamics of the rotating magnetized neutron star. The analysis of the radiation time series in this paper is intended to test the fundamental emission mechanism on the timescale of an individual burst and does not model directly the ensemble emission from the polar cap radio source.

\section{DESCRIPTION OF PLASMA WAVE TURBULENCE IN THE PULSAR MAGNETOSPHERE}

This section summarizes the evolution of plasma wave turbulence as derived from a computer solution to the nonlinear coupled wave equations.

The turbulence is presumed to originate with a plasma two-stream instability (Cheng \& Ruderman 1977). A single wavemode, $(m, n)=(9,0)$, with $k_{m n}=0.9 \omega_{p} / c$ is incremented in the computer solution with each time step at a rate $v_{\text {growth }}=0.001 \omega_{p}$ to simulate a beam-plasma instability. This produces a growing plasma wave propagating in the magnetic field direction.

At large amplitudes, the beam-resonant mode becomes modulationally unstable to transverse wavenumber $k_{\perp}=$ $\left(\omega_{p} / c\right)(\Lambda / 2)^{1 / 2} . \Lambda$ is a dimensionless nonlinear parameter defined as

$$
\Lambda=E^{2} /\left(8 \pi n_{0} m \bar{v}_{\|}^{2}\right)
$$

As the nonlinear interaction intensifies, the instability produces filamentation of the initial beam-resonant wave, followed by "collapse" of the waves along the magnetic field as wave energy increasingly accumulates in spatially localized regions. This phenomenon is characteristic of strong plasma turbulence in unmagnetized electron-baryon plasmas (Zakharov 1972; Goldman 1984). An additional nonlinear phenomenon revealed by the numerical solution is the recurrent behavior in which part of the system energy is transferred back and forth between the nonlinear state and the initial state (Yuen \& Ferguson 1978). This has the significant effect of modulating wavemode amplitudes.

The turbulent cascade develops very quickly. The dynamical nonlinear timescale for modulational instability, recurrence, and collapse, are all governed by similar rates (given here as the maximum of the modulational instability growth):

$$
v_{\mathrm{NL}} \sim \omega_{p} \Lambda / 2 .
$$

Modulational instability and strong turbulence have important consequences for radio emission because they generate superluminal waves with finite Poynting flux that can escape from the plasma as radiation.

The radiative loss also affects the turbulent energy density. The basic radiative loss rate is

$$
v_{\text {loss }}=c / L
$$

where $L$ is the size of the source region. This dissipation is modeled in the computer code by damping each mode at a rate proportional to its Poynting flux: $v\left(k_{m}, k_{n}\right)=$ $-v_{\text {loss }}\left[1-\left(\hat{\boldsymbol{e}}_{m n} \cdot \boldsymbol{k}_{m n}\right)^{2} /\left|\boldsymbol{k}_{m n}\right|^{2}\right]^{1 / 2}$.

Because the turbulent cascade is driven at the expense of energy in the beam-resonant mode, the onset of turbulence also has the effect of diminishing the rate at which energy is added to the beam-resonant mode. Therefore, the turbulence is nonsteady, and the radio emission is intermittent.

The numerical solutions thus support the description of pulsar radio emission as a nonlinear transition to strong plasma turbulence, which follows a slow buildup of electrostatic wave energy in plasma waves, where modulational instability and strong turbulence are mechanisms for the mode conversion into electromagnetic waves.

\section{MODULATIONAL INSTABILITY}

There are a number of reasons why modulational instability (Nishikawa \& Liu 1976; Hasegawa 1970) plays an important role in the nonlinear physics of the turbulence. As noted in $\S 3$, modulational instability initiates the transition from the electrostatic instability to the turbulent state. In this section, it will be shown that the modulationally stimulated waves are prominent in the turbulent wave spectrum and therefore contribute substantially to the escaping radiation. The unique temporal signature produced by the evolution of the coherent modulational interaction will also be examined.

Modulational instability can be described as an oscillating two-stream instability (Bardwell \& Goldman 1976) involving the interaction of four waves: two electrostatic pump waves with the wavenumber of the beam-driven wave, and two oppositely propagating growing waves, as shown in Figure 1.

The turbulent wave spectrum that is derived from the numerical solution is shown as a contour plot of mode amplitude in Figure 2. The wave spectrum is dominated by 


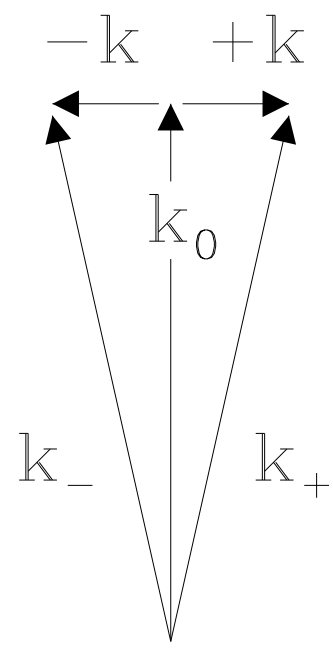

FIG. 1.-Momentum conservation in the modulational instability. The growing modes, $k_{+}=k_{0}+k$ and $k_{-}=k_{0}-k$, beat resonantly with the pump mode, $k_{0}$, through coupling with a transverse perturbation, $k$.

the pump mode, $(m, n)=(9,0)$, and the modulationally stimulated modes at $(m, n)=(9,1)$ and $(9,-1)$. The selffocusing phenomenon described as wave collapse is a multimode modulational excitation that broadens the wave spectrum (Vedenov \& Rudakov 1964); this is also apparent in Figure 2. The analysis of radiative characteristics (which follows in $\S 6$ ) focuses on the excitation of the mode $(9,1)$, which is identified by the wavevector $\boldsymbol{k}_{+}$in Figure 2.

As the instability saturates, the beam-driven and modulational modes undergo oscillations in amplitude on a timescale distinct from the rate of growth. This is a manifestation of a nonlinear recurrence phenomenon that was discovered in context of the nonlinear Schrödinger

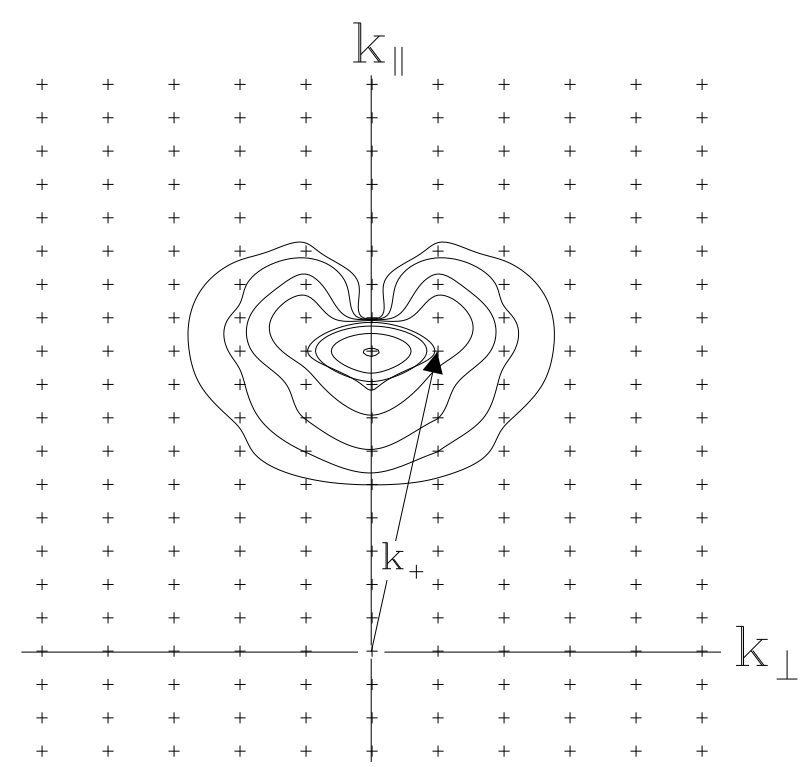

FIG. 2.-Contours of squared wave amplitude in wavenumber space showing the spectral distribution of developed turbulence. The $i$ th contour level is $A_{\max }^{2} / 2^{i}$, where the maximum at the pump mode, $(m, n)=(9,0)$, has the value $A_{\max }^{2}=0.04\left(8 \pi m n \bar{v}_{\|}^{2}\right)$. The wavevector $k_{+}$at $(m, n)=(9,1)$ corresponds to a radiative mode initiated by the modulational instability. The wavenumber spacing parallel to the magnetic field is $\omega_{p} / c$, and the perpendicular spacing is $2 \omega_{p} / c$. equation by Yuen \& Ferguson (1978). In plasma wave recurrence, the initial growth of the unstable modulation of a spatially uniform large-amplitude wave saturates, and the wave excitation reverts to the initial uniform state. Thyagaraja (1979) compared this phenomenon to Poincare recurrence of bounded Hamiltonian systems, in which the low number of degrees of freedom is important. In the Schrödinger wave system, the degrees of freedom correspond to distinct wavemodes. Using conserved quantities of the nonlinear Schrödinger equation, such as wave energy, Thyagaraja (1979) shows that the nonlinear transfer of wave energy to shorter spatial scales and larger wavenumbers must be limited to a finite number of degrees of freedom. Therefore, the redistribution of energy is among a finite set of modes, and it follows that turbulence described by the cubically nonlinear Schrödinger equation can show recurrence. The presence of dissipation by radiative loss in the present system serves to further inhibit the degrees of freedom.

A heuristic description of this behavior can be provided by an analog to coupled pendulums. We regard three oscillators: the pump mode, $A_{0}$, and the modulational modes $A_{+}$and $A_{-}$. Recognizing that the coupling between the modes is intrinsically nonlinear, with coefficients $\epsilon_{+}=\omega_{p}^{2} A_{0}^{*} A_{+}, \quad \epsilon_{-}=\omega_{p}^{2} A_{0}^{*} A_{-}, \quad K_{+}=\left(\omega_{+}^{2}-\omega_{p}^{2}\right)+$ $\omega_{p}^{2}\left|A_{0}\right|^{2}+\omega_{p}^{2}\left|A_{-}\right|^{2}, \quad K_{0}=\left(\omega_{0}^{2}-\omega_{p}^{2}\right)+\omega_{p}^{2}\left|A_{+}\right|^{2}+$ $\omega_{p}^{2}\left|A_{-}\right|^{2}$, and $K_{-}=\left(\omega_{-}^{2}-\omega_{p}^{2}\right)+\omega_{p}^{2}\left|A_{+}\right|^{2}+\omega_{p}^{2}\left|A_{-}\right|^{2}$, a useful model can be constructed from equation 2 in a form suggestive of coupled mode oscillators:

$$
\omega \omega_{p}\left(\begin{array}{c}
A_{+} \\
A_{0} \\
A_{-}
\end{array}\right)=-\left(\begin{array}{ccc}
K_{+} & \epsilon_{-}^{*} & 0 \\
\epsilon_{-} & K_{0} & \epsilon_{+} \\
0 & \epsilon_{+}^{*} & K_{-}
\end{array}\right)\left(\begin{array}{c}
A_{+} \\
A_{0} \\
A_{-}
\end{array}\right) .
$$

Behavior of the modulated amplitude can be demonstrated by replacing the nonlinear coefficients by values of $K \sim$ $K_{+} \sim K_{0} \sim K_{-}$, and wave modulation $\epsilon \sim \epsilon_{+} \sim \epsilon_{-}$. For numerical calculation, the wave energy density parameter $\Lambda \omega_{p}^{2}$ is used for $K$; for a fully modulated wave, $\epsilon \sim \frac{1}{6} \Lambda \omega_{p}^{2}$ is used. Solution of the simplified eigenvalue equation shows that the energy transfers between the pump $A_{0}$ and side modes $A_{+}$and $A_{-}$at the frequency $\omega_{1}-\omega_{2}$, where the frequencies are

$$
\omega_{1,2}=\omega_{p}-\Lambda \omega_{p} \pm \frac{1}{\sqrt{18}} \Lambda \omega_{p} .
$$

Despite the somewhat arbitrary construction of the coupled oscillator model, the two frequencies do, in fact, appear in the frequency spectrum of the turbulence. Because the separation of the frequencies depends on the in situ amplitude of the turbulence, this suggests an interesting observational diagnostic.

Generally, the recurrent motion is not strictly periodic and not limited to the two frequencies displayed above. However, strong dissipation contributes to the narrow frequency spectrum in this case. Thyagaraja (1981) develops an analytic formula for the recurrence rate in the form of a Rayleigh quotient, $Q(t)$, involving invariant functionals of the (simple) cubically nonlinear Schrödinger equation. This formula bounding the recurrence time resembles the one inferred from equation (8).

In addition to the modulational instability, other mode transfer processes have been proposed in connection with pulsar emission. In the model by Beskin, Gurevich, \& Iso- 
tomin (1988), a curvature-plasma mode instability takes the place of the plasma two-stream Langmuir-mode instability and three-mode coupling takes the place of four-mode modulational coupling in the conversion. The recurrent behavior described here may distinguish between these types of mode transfer radiation mechanisms, depending on whether the three-wave decay process also exhibits recurrent behavior. If, for example, the wave decay process produces a spectral cascade (weak turbulence), this type of nonlinear behavior could produce different observable features from four-wave modulational evolution toward spatially coherent wave collapse (strong turbulence). A defining property of the underlying wave turbulence may be whether it exhibits deterministic chaotic behavior, since recurrence is a behavior associated with chaotic systems. An analysis of the turbulent conversion radiation model finds, in fact, a strange attractor (Delaney 1998).

\section{TURBULENT CONVERSION AS AN EMISSION MECHANISM}

The utility of turbulent conversion for the theory of pulsar emission can be illustrated with the following numerical example. The computer solutions show that the nonlinear transition occurs when the nonlinear parameter is $0.1 \lesssim \Lambda \lesssim 1$. Other source parameters need to be estimated. For example, one can assume that the plasma size is of the order of the pulsar polar cap, $L=R_{*}\left(\Omega_{*} R_{*} / c\right)^{1 / 2}$, where $R_{*} \sim 10^{6} \mathrm{~cm}$ is the neutron star radius and $\Omega_{*}$ is the rotation frequency. Because the internal energy might be expected to be relativistic or nearly relativistic, let $k_{\mathrm{B}} T$ $\sim m c^{2}$. In order to infer a local plasma frequency from the Doppler-boosted radio signal, $v_{p}=v / 2 \gamma$, the bulk plasma velocity is estimated to have a relativistic factor $\gamma \sim 300$. With these inferred and estimated parameters, the turbulent energy density in the magnetospheric source plasma of a $0.033 \mathrm{~s}$ period pulsar (using the Crab pulsar as an example) can be calculated to be

$$
\frac{E^{2}}{8 \pi} \sim n m \bar{v}_{\|}^{2}=0.2 \mathrm{ergs} \mathrm{cm}^{-3} .
$$

If this energy is released into radiation on the timescale of the light crossing time (also the dissipation rate), $L / c$, the power in the plasma rest frame is $d P^{\prime} / d \Omega^{\prime}=2.6 \times 10^{18} \mathrm{ergs}$ $\mathrm{s}^{-1}$. The power received by a stationary observer in the relativistically boosted radiation beam is $d P / d \Omega=(2 \gamma)^{4} d P^{\prime} /$ $d \Omega^{\prime}$ (Rybicki \& Lightman 1979), and the received flux is $(d P / d \Omega) /\left(R^{2} \Delta v\right)$. The intrinsic bandwidth is not known, but $\Delta v \sim 0.1 v$ in the computer solutions (described in $\S 6$ ). From the distance of the Crab pulsar, $R=6 \times 10^{21} \mathrm{~cm}$, the computed flux is

$$
\begin{aligned}
F_{v}= & 0.2 \mathrm{Jy}\left(\frac{L}{8 \times 10^{4} \mathrm{~cm}}\right)^{2}\left(\frac{k_{\mathrm{B}} T}{500 \mathrm{keV}}\right) \\
& \times\left(\frac{v_{p}}{5 \mathrm{GHz} / 300}\right)^{2}\left(\frac{\gamma}{300}\right)^{4}\left(\frac{500 \mathrm{MHz}}{\Delta v}\right)\left(\frac{\Lambda}{0.1}\right),
\end{aligned}
$$

as is quite reasonable allowing for the uncertainties in the model parameters. Typical pulsed radio flux from the Crab is slightly less than $1 \mathrm{Jy}$, with giant peaks up to $10^{4} \mathrm{Jy}$ (Lundgren et al. 1995).

By invoking radius-to-frequency mapping, a crude frequency spectrum can be inferred from equation (10). The following dependences are assumed: constant velocity current flow along magnetic field lines, so $L \propto r^{3 / 2}$ and $v_{p} \propto r^{-3 / 2}$; a burst bandwidth proportional to the nonlinear frequency shift, $\Delta v \propto \Lambda v$ (see, for instance, eq. [8]); and constant temperature in the flow. With these assumptions, the flux is predicted to fall off as

$$
F_{v} \propto v^{-1} \text {. }
$$

Not included in this simple scaling are the statistics of superimposed bursts or details relating to the structure of the polar cap current flow.

\section{SPECTRAL SIGNATURES OF TURBULENT EMISSION}

The very short-time emission behavior is of particular interest, since it is closely tied to the physics of the emission process. Computer solutions of turbulent emission show that the time structure of a single pulse is governed by the two timescales given by $v_{\mathrm{NL}}^{-1}$ and $v_{\text {loss }}^{-1}$, i.e., the nonlinear dynamical time and the radiative loss time. Note that the growth time is largely decoupled from the details of the emission, except for the buildup of the turbulence and possible connection with the longer-timescale microstructure.

The dynamical timescale of the turbulence (eq. [5]) depends on the value of $\Lambda$ when the turbulence decouples from the beam-driven wave. Simulations show this to be consistently on the order of 0.1 , so the characteristic structure will be tens of wave periods long. At $5 \mathrm{GHz}$, this corresponds to about 1 ns or less.

The radiation loss time (eq. [6]) in this numerical model is also the time during which the system maintains its coherence. In the case of weak dissipation, the coherence timescale is longer than the dynamical timescale. As a result, a single pulse is long enough to exhibit modulation owing to the recurrence effect described in Section $\S 4$. The burst continues until the turbulent energy is diminished by the dissipative loss.

An example of this characteristic pulse structure is shown in Figure 3. The radiation loss parameter is $v_{\text {loss }}=0.05 \omega_{p}$; therefore, the dissipation timescale is on the same order as the nonlinear timescale. $E(t)$ is the electric field waveform derived from the in situ amplitude of the mode $(m, n)=$ $(9,1)$. In addition to the time series of wave electric field, Figure 3 shows the power spectrum and two inferred observational functions: the amplitude-squared detection, and the autocorrelation function.

The power spectrum exhibits the two frequencies identified in equation (8). From the displacement from the plasma frequency and the separation of the two frequencies, the nonlinear plasma parameter is inferred to be $\Lambda \sim 0.1$, which is consistent with the actual excitation of the turbulence.

The third frame shows an example of a detected signal. If the wave electric field at frequency $v_{0}$ is given by $E(t)=$ $a(t) \exp \left(i v_{0} t\right)$, so that $a(t)$ is the amplitude of a signal, the amplitude-squared detection is proportional to $I(t)=|a(t)|^{2}$. Before computing $a(t)$, the spectral field $E(v)$ is multiplied by a cosine filter spectral function $S(v)$ given by $S(v)=\cos \left[\pi\left(v-v_{0}\right) / \Delta v\right]$ within the frequency band $v_{0}-\Delta v<v<v_{0}+\Delta v$ and by $S(v)=0$ outside band. This detection function constructs a received signal at frequency $v_{0}$ through a bandpass of $\Delta v$, as it would be recorded by radio telescope observation. The vertical lines on the power spectrum represent the bandpass of the filter used in the calculation. Note that the bandpass necessary to detect the 

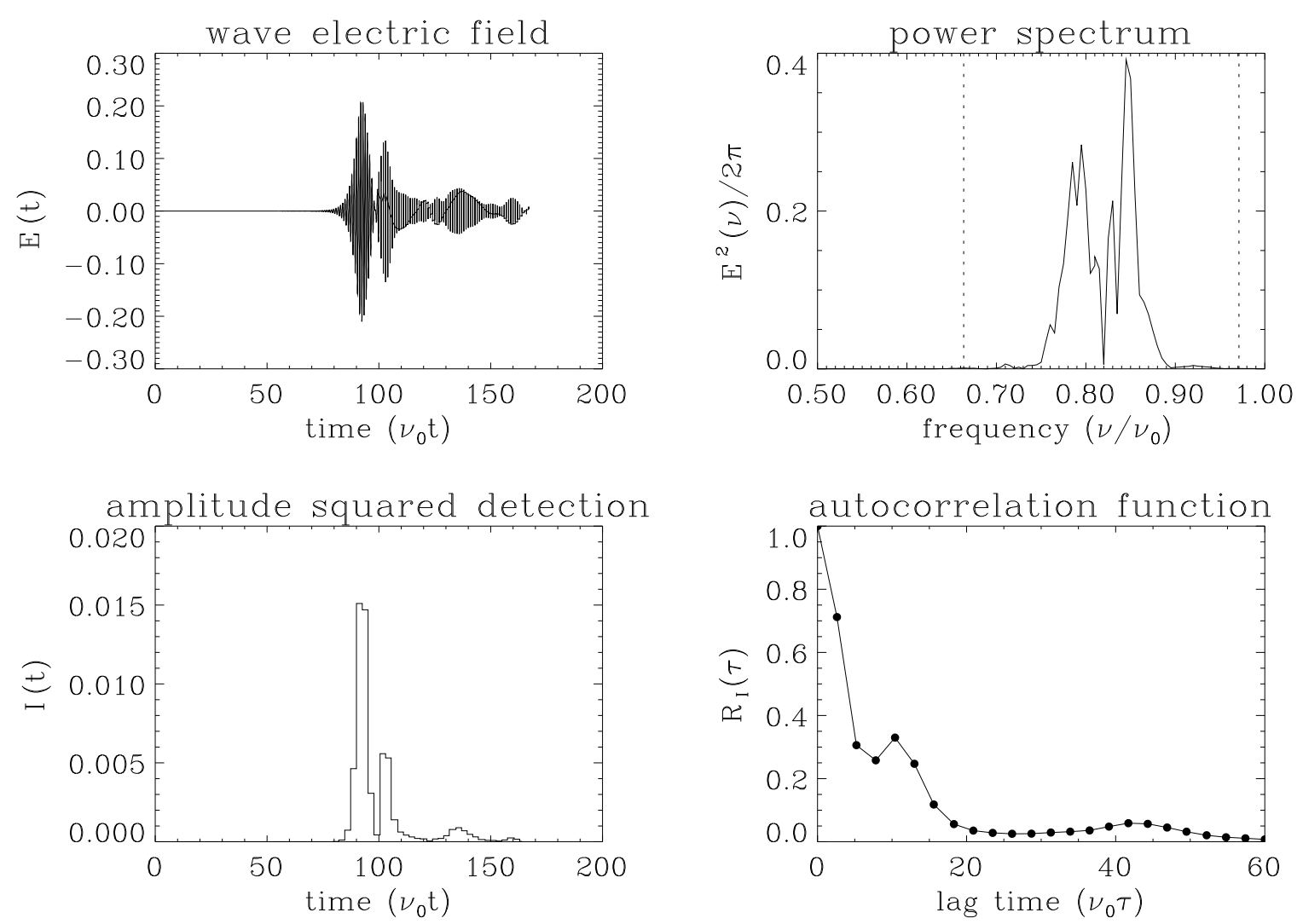

FIG. 3.-Numerical simulations of a nanopulse. Time is given in units of $v_{0}=2 \gamma v_{p}$, where $v_{p}$ is the plasma frequency in the plasma frame. Then $v_{0} t$ is the number of wave periods in the observer's frame. Upper left: amplitude of the electric field in the mode $(m, n)=(9,1)$ normalized to $\left(8 \pi n m \bar{v}_{\|}^{2}\right)^{1 / 2}$ corresponding to the emitted signal; Upper right: Power spectrum of a nanopulse; Lower left: Total intensity of a nanopulse as it would be detected by a radio telescope observation at $v_{0}$, band-limited to $0.1 v_{0}$ and decimated to a sampling rate of $2.5 v_{0}^{-1}$. Lower right: Autocorrelation function of the pulse intensity, as it would be observed with these instrumental parameters.

modulated signal at $5 \mathrm{GHz}$ is several hundred $\mathrm{MHz}$. The nominal received frequency $v_{0}$ is assumed to be related to the local pulsar plasma frequency by the Lorentz shift $v_{0} \sim$ $2 \gamma v_{p}$, where $\gamma$ is the Lorentz factor of the motion of the pulsar current flow in the laboratory frame. The spectral analysis does not depend directly on assumed values of $\omega_{p}$ and $\gamma$ because the simulation scales to $v_{0}$. The intrinsic temporal pulse width, the nonlinear recurrence time, and the coherence time all scale to the observation frequency as $v_{0}^{-1}$ : thus, the nanostructure will broaden with lower frequency.

The autocorrelation function is computed by multiplying the Fourier transform of intensity by itself, and transforming the product back into the time domain: $R_{I}(\tau)=$ $F T\left[I^{2}(\omega)\right] / \bar{I}^{2}(t)$. The autocorrelation function of the pulsar signal is calculated in this fashion and is employed by observers to analyze the temporal structure of radio data (see, e.g., Cordes 1976). The fourth frame in Figure 3 shows a secondary peak in the autocorrelation function due to the nonlinear recurrence in the underlying turbulence. Other peaks that appear can be aperiodic, since recurrence is a chaotic process. This autocorrelation function applies to the signal of a single burst on extremely short lag times.

The distinction between what is known as microstructure and the "nanostructure" described in this paper is made deliberately. Nanostructure is not merely microstructure resolved on a shorter timescale. Nanostructure is the intrinsic structure of an individual burst, which in pulsar phenomenology might be referred to as a "shot." The pulsar signal is modeled as shot noise (Cordes 1976), namely, a random superposition of individual bursts. The modulation of the amplitude of the shot noise is the interpretation applied to the microstructure autocorrelation function (Rickett 1975). Nonlinear wave dynamics determines the nanostructure. The existence of microstructure implies a systemic change in the amplitude of burst events, influenced, perhaps, by modulation in the polar cap current flow, or in other behaviors of the turbulence; for example, nucleation of the spatial wave collapse (Doolen, DuBois, \& Rose 1985; DuBois, Rose, \& Russell 1988). Microstructure is seen in pulsars only at low radio frequency (below $\sim 1$ $\mathrm{GHz}$ ), while nanostructure should be present at all frequencies.

\section{SUMMARY}

This analysis shows that the emission microphysics can leave imprints on the received signal. Here, specific temporal behaviors are inferred from the theory of turbulent plasma emission. The derived autocorrelation function associated with nanostructure in an individual resolved pulse is depicted schematically in Figure 4. The model predicts several characteristic features. First, there is an intrinsic pulse width of about one nanosecond, which is governed by the timescale of the modulationally induced turbulent collapse, as given in equation (5). Second, the nanostructure indicated by peak(s) in the autocorrelation function is due 


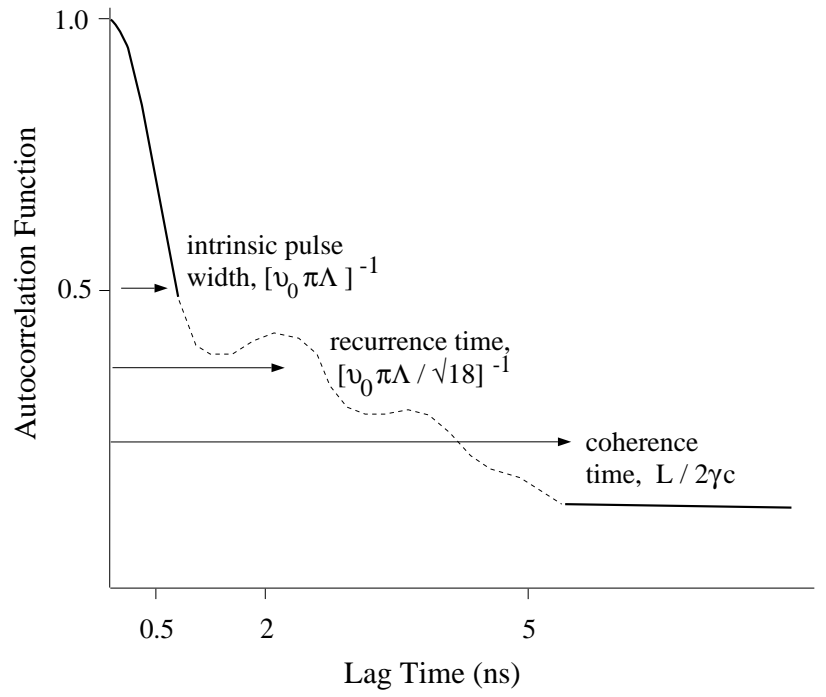

FIG. 4.-Characteristic autocorrelation function at $5 \mathrm{GHz}$ showing nanostructure in emission derived from plasma turbulence stimulated by the modulational instability. Characteristic timescales are identified assuming the nonlinear parameter $\Lambda=0.1$ and source size $L$, given by the polar cap size of the Crab pulsar.

to the coupling between the radiative mode and the plasma mode, with the recurrence timescale given by $2 \pi /\left(\omega_{1}-\omega_{2}\right)$, where the two frequencies are given in equation (8). Third, the intrinsic nanostructure extends to almost $10 \mathrm{~ns}$, as determined by the coherence time. The coherence time, set by the radiation loss rate, is estimated from the light travel time across the source volume, as in equation (6). Note that the nanostructure will be present at all frequencies. The characteristic timescales are frequency dependent, becoming longer at lower frequency.

The turbulence emission model can be tested using high time resolution observations of pulsar radiation. In general terms, the emission model suggests intrinsic pulse widths smaller than $1 \mathrm{~ns}$, which may repeat over timescales of a few nanoseconds to $10 \mathrm{~ns}$. The theory predicts spectral sidebands whose separation in frequency is a function of the nonlinearity of the medium (i.e., the in situ electric field amplitude). Based on the predictions, an observational bandwidth of $10 \%$ might be necessary to detect the spectral signature. Such structure, if observed, would relate directly to parameters in the source region, specifically source size and turbulent energy density.

In conclusion, the observational detection of multiplehumped correlation functions on these very short timescales would strongly support an emission mechanism involving mode conversion transfer effects, such as are documented here in the conversion of plasma wave turbulence.

In addition to the theory in this paper, mode conversion is invoked in a broad class of collective stimulated scattering processes proposed for pulsars, including masers. Analysis of other mode conversion mechanisms will be needed to determine whether the spectral signature is unique to the modulational transfer mechanism.

My thanks to Tim Hankins, Jean Eilek, Dave Moffet, Phil Hume, Tracey Delaney, and Paul Arendt for useful and interesting discussions, and for support from NSF grant AST 93-15285.

\section{REFERENCES}

Arons, J. 1981, in IAU Symp. 95, Pulsars, ed. W. Sieber \& R. Wielebinski (Boston: Reidel), 69

Arons, J., \& Barnard, J. J. 1986, ApJ, 302, 120

Asseo, E. 1993, MNRAS, 264, 940

1996, in IAU Colloq. 160, Pulsars: Problems and Progress, ed.

S. Johnston, M. A. Walker, \& M. Bailes (San Francisco: ASP), 147

Asseo, E., Pelletier, G., \& Sol, H. 1990, MNRAS, 247, 529

Bardwell, S., \& Goldman, M. V. 1976, ApJ, 209, 912

Beskin, V. S., Gurevich, A. V., \& Isotomin, Ya. N. 1988, Ap\&SS, 146, 205

Cheng, A. F., \& Ruderman, M. A. 1977, ApJ, 212, 800

Chian, A. C.-L. 1992, in IAU Colloq. 128, The Magnetospheric Structure and Emission Mechanisms of Radio Pulsars, ed. T. H. Hankins et al. (Zielona Gora, Poland: Pedagogical Univ. Press), 356

Chian, A. C.-L., \& Kennel, C. F. 1983, Ap\&SS, 97, 9

Cordes, J. M. 1976, ApJ, 208, 944

Delaney, T. 1998, M.S. thesis, New Mexico Inst. of Mining and Tech.

Doolen, G. D., DuBois, D. F., \& Rose, H. A. 1985, Phys. Rev. Lett., 54, 804

DuBois, D. F., Rose, H. A., \& Russell, D. 1988, Phys. Rev. Lett., 61, 2209

Gangadhara, R. T., Krishan, V., \& Shukla, P. K. 1993, MNRAS, 262, 151

Goldman, M. V. 1984, Rev. Mod. Phys., 56, 709

Hankins, T. H., \& Weatherall, J. C. 1998, in Proc. 18th Symp. on Relativistic Astrophysics and Cosmology, ed. Olinto, A. V., Frieman, J. A., \& Schramm, D. N. (New Jersey: World Scientific)
Hasegawa, A. 1970, Phys. Rev. A, 1, 1746

Lundgren, S. C., Cordes, J. M., Ulmer, M., Matz, S. M., Lomatch, S., Foster, R. S., \& Hankins, T. 1995, ApJ, 453, 433

Melrose, D. B. 1996, in IAU Colloq. 160, Pulsars: Problems and Progress, ed. S. Johnston, M. A. Walker, \& M. Bailes (San Francisco: ASP), 139

Moffet, D.A. 1997, Ph.D. thesis, New Mexico Inst. of Tech.

Mofiz, U. A., de Angelis, U., \& Forlani, A. 1985, Phys. Rev. A, 31, 951

Nishikawa, K., \& Liu, C. S. 1976, in Advances in Plasma Physics, Vol. 6, ed. A. Simon \& W. B. Thompson (New York: Wiley)

Rickett, B. J. 1975, ApJ, 197, 185

Rybicki, G. B., \& Lightman, A. P. 1979, Radiative Processes in Astrophysics (New York: Wiley), 141

Thyagaraja, A. 1979, Phys. Fluids, 22, 2093

. 1981, Phys. Fluids, 24, 1973

Vedenov, A. A., \& Rudakov, L. I. 1964, Dokl. Akad. Nauk SSSR, 159, 767 [Sov. Phys. Dokl. 9, $1073(1965)]$

Weatherall, J. C. 1997, ApJ, 483, 402

Yuen, H. C., \& Ferguson, W. E. 1978, Phys. Fluids, 21, 1275

Zakharov, V. E. 1972, Zh. Eksp. Teor. Fiz., 62, 1745 [Sov. Phys. JETP, 35, 908 (1972)] 